\title{
The Reconsider Linear Non-Threshold (LNT) Hypothesis of Radiation Effect on Living Object
}

\author{
Osaka University, Yuichiro Manabe \\ Chinese Academy of Science, Issei Nakamura \\ Osaka University, Hiroo Nakajima \\ Kyoto University, Yuichi Tsunoyama \\ NPO Einstein, Masako Bando
}

\begin{abstract}
After Muller's experiment on drosophila, it was found that the risk of radiation depends on the total dose, and the linear-non-threshold (LNT) hypothesis became the basis of radiation protection. However, Russel et al. later showed the existence of the dose rate effect. We propose a mathematical model for integrating the results for different species and extrapolating them to humans. With scaling rule, the model can help interpret various data in a comprehensive manner. This would produce new knowledge in radiation risk assessment.
\end{abstract}

\section{Introduction}

The discovery of mutations by artificial radiation (Muller's experiment on drosophila) led to the conclusion that "The mutation rate is proportional to the total dose of irradiated radiation and that the proportional coefficient does not change for different dose rates (Linear-Non-Threshold, LNT)" " ${ }^{1)}$. It is understandable that the scientists at that time thought this way since it only requires the dose $(D)$ that causes mutation and the amount of mutation is a physical process. After this discovery, radiation protection was based on the LNT hypothesis.

Later, however, an experiment with 7 million mice at Oak Ridge National Laboratory (Russel's mega-mouse project) led to the discovery that the mutation rate changes with the dose rate ${ }^{2}$. Figure 1 shows the results. If there was thorough analysis of the implication of the mega-mouse project, the dose rate standard would have replaced the LNT hypothesis and the protection standards might have been corrected.

The important question is how the output, balanced with the input (determined by the dose rate), is different when the input is different. If the input and output are not related to the amount accumulated in the body, the balance is determined by the difference between the input and the output. However, if the output is proportional to the accumulated amount,

DOI : 10.15669/fukushimainsights.Vol.1.278

(C) 2021 Atomic Energy Society of Japan. All rights reserved.

Originally published in Journal of the Atomic Energy Society of Japan (ISSN 1882-2606), Vol. 56, No. 11, p. 705-708

(2014) in Japanese. (Japanese version accepted: August 20, 2014) 
certain accumulation will not allow further accumulation even with more input. Therefore, it is important to observe the change with time. Unfortunately, however, the impact of LNT hypothesis obtained by Muller's experiment persists, leading to a fixation on the idea that mutation (mutation frequency) depends on total dose, and the sole importance is placed on total dose. Therefore, Russel's discovery that the mutation rate drastically changes with low dose rates even with same total dose was considered to supplement the LNT hypothesis.

In fact, Russel also concluded that the slope of LNT is different between low dose rates and high dose rates and semi-forcefully explained the experiment data with two lines, introducing the ratio of the slopes as the Dose and Dose Rate Effectiveness Factor (DDREF) (See Figure 1).

Let us examine the details of Russel's data in Figure 1. The horizontal axis is the total dose, and the vertical axis is the mutation frequency. A rough idea of the relationship between the dose and the mutation frequency can be obtained since the plot has experimental data for Drosophilinae and Tradescantia are provided, which are well-referenced. With the same total dose, the difference between the slopes of mutation frequency for acute dose $\left(\sim 10^{1}(\mathrm{~Gy} / \mathrm{hr})\right)$ and chronic dose $\left(\sim 10^{-4}-10^{-3}(\mathrm{~Gy} / \mathrm{hr})\right)$ is larger than that between the errors, failing the explanation for experimental data with a single line. However, there has not been a formulation to quantitatively evaluate this difference in the dose rate.

Consider pouring water in a cylinder. When pouring $1 \mathrm{cc}$ of water per second, the total water accumulated in the cylinder matches the total water poured; however, if the cylinder has a hole at the bottom, water will not accumulate unless the input rate is higher than the output rate through the hole. With an increase in the pouring rate, water is accumulated. However, the water pressure increases, thereby increasing the output rate. Finally, the input rate and output rate are balanced, leading to constant accumulation of water. From this point onwards, the accumulated water will remain constant even if one continues to pour water. Members of the Atomic Energy Society is familiar with this phenomenon since it is the same as the concept that exposure does not keep accumulating when absorbing $1 \mathrm{~Bq}$ of radiation per day through food in Fukushima. Radioactive elements have two decays (consider these as outputs), a physical half-life and a biological half-life, and they decrease at a certain rate. For this reason, daily intake of a certain amount of radiation from radionuclides is balanced with the output, leaving the exposure constant. There are numerous examples of such balances in

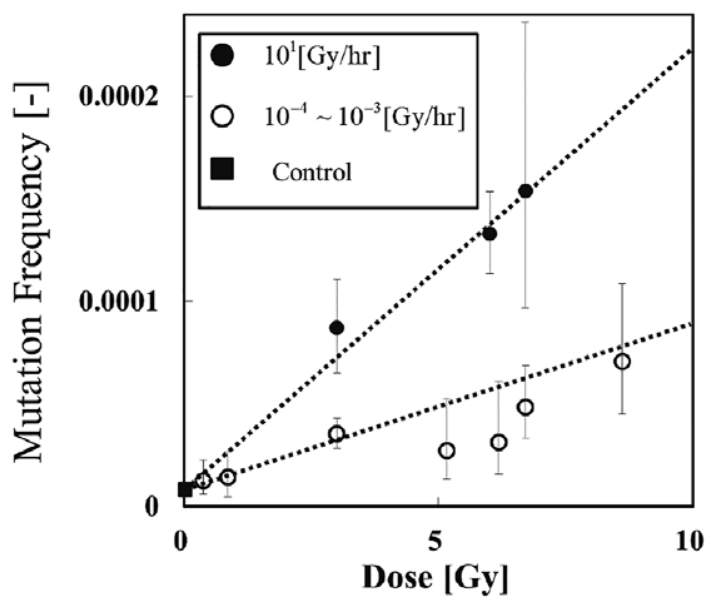

Figure 1 Experimental results of Russel's mega-mouse project 
natural phenomena, e.g., in the ozone layer, ozone is decomposed at the bottom of the layer while it is created at the top of the layer. Although it appears to have a constant thickness, the input and output are dynamically balanced.

\section{From Lea Target Theory to WAM Model}

From bacteria to humans, organisms are diverse, but the smallest unit of living beings is cells; multi-cell systems have common structures, in which they adapt to the environment and continue the cycle of metabolism and multiplication. Biologists are most interested in diversity, while physicists are interested in the unified picture and quantitativeness.

Let us consider a certain organ in a living organism as an n-body system composed of normal cells and mutated cells. Cells respond to external micro-stimuli, $\Delta$, in various ways. The changes in mutated and normal cells are mathematically expressed as follows.

$$
\begin{aligned}
\Delta N_{n} & \Leftrightarrow\left(T_{n n} N_{n}+T_{n m} N_{m}\right) \times \Delta \\
\Delta N_{m} & \Leftrightarrow\left(T_{m n} N_{n}+T_{m m} N\right) \times \Delta
\end{aligned}
$$

Here, $N_{n}$ and $N_{m}$ are the numbers of normal and mutated cells in the system, respectively. There is a significant difference between choosing time $(t)$ and dose $(D)$ for micro-variation. In the 1950s, Lea proposed the "target theory," which reproduces Muller's experimental results and provides a fundamental formulation of radiation biology ${ }^{3)}$. The formulation was based on the following differential equations,

$$
d N_{n}=-N_{n} \frac{d D}{D_{0}} \rightarrow N_{n}(D)=N_{0} e^{-\frac{D}{D_{0}}}
$$

In Lea's formulation, let us consider the simplest case, a 1-target-1-hit case. $D_{0}$ is the average lethal dose necessary for hitting the target (most receptive part of the cell). When the normal cells are dominant in the system, the mutation frequency $F$ is

$$
F(D)=\frac{N_{m}}{N_{0}}=\frac{N_{0}-N_{n}}{N_{0}}=1-e^{-\frac{D}{D_{0}}}
$$

As a physical process, this equation is consistent but neglects the contributions from an organism's repairing process, apoptosis, and mutant-cell death. Later, researchers made corrections, but the tradition of taking dose $D$ as the variable continued ${ }^{4,5)}$.

According to the description in Chapter I, time is intimately related to the repairing process. Here, we develop our work and consider the following equation ${ }^{6}$.

$$
\frac{d}{d t} F(t)=(\kappa+\gamma d)-(\alpha+\beta d) F(t)
$$

Here, $d$ is the dose rate. The first term is the mutation rate of normal cells, and the second term corresponds to the repairing process, which tends to reduce $F$. Note that each term includes parameters proportional to the dose rate (stimulation) as well as $\kappa$ and $\alpha$. This is because mutation of cells and repairing functions are present even without artificial irradiation. If $d$ is constant with time, the solution can be easily obtained, which is 


$$
\begin{gathered}
F(t)=F(\infty)\left(1-\mathrm{e}^{-(\alpha+\beta d) t}\right)+F(0) \mathrm{e}^{-(\alpha+\beta d) t}, \\
F(\infty)=\frac{\kappa+\gamma d}{\alpha+\beta d}
\end{gathered}
$$

This familiar form is a special form of the Richards differential equation, known as growth curve $^{7)}$

$$
\frac{d}{d t} W(t)=\eta\{W(t)\}^{m}-k W(t)
$$

In other words, our equation corresponds to Eq. (6), where

$$
m=0, W(t)=F(t), \eta=\kappa+\gamma d, k=\alpha+\beta d
$$

$m$ in Eq. (6) is called the geometry parameter, which stops the growth when the growth reaches the maximum. If $m=0$, the curve is a Logistic curve, and $m=1$ corresponds to a Gompertz curve. This is often used as a growth model for animals ${ }^{1}$. The growth curve is not a simple increasing function, and the growth slows down as $k t$ becomes of order 1 and $W$ approaches $W(\infty)$ as time passes.

Equation (5) reveals that the parameters such as $\eta$ and $k$ in (6) depend on external stimulation (radiation), i.e., the dose rate, and indicate that they explicitly regulate growth in response to stimulation, which is the feature of our formula. This term explains the difference in mutation frequency induced by the difference in the dose rate.

For the sake of comparison with the experiments, we devise the formulation so that species can be analyzed in a unified manner. We rearrange the solution of $F$ in (5) and define a screening function common to organisms.

$$
\Phi(\tau) \equiv \frac{F(t)-F(0)}{F(\infty)-F(0)}=1-\mathrm{e}^{-\tau}, \tau \equiv(\alpha+\beta d) t
$$

$\tau$ is the dimensionless time, and when this becomes of order 1 , restoration function and input are balanced and converge to a certain value with no dependence on species. We call the above model the Whack-A-Mole (WAM) model. The naming comes from the observation that organisms "whack" mutated cells in response to stimulation.

\section{Comparison with Experimental Data}

There are 4 parameters $(\kappa, \gamma, \alpha$, and $\beta)$ in the WAM model. The experimental data show the mutation frequency for irradiation with varying dose rates. Most studies in literature have given attention to the total dose, and only a few studies have emphasized the dose rate. Among those few studies, we applied our mathematical model to the data of five species of animals (mice and drosophila) and plants (maize, Tradescantia, and chrysanthemum) ${ }^{2,8-11)}$. With experimental subjects and the irradiation rate (assumed to be constant), we determined the 4 parameters via an $\chi^{2}$ test, convert data with different dose rates and the total dose to scaling functions, and compared with the experimental data. Obviously, sensitivity and recovery effect against radiation, reflected in the 4 parameters, vary depending on species, sex, and type of organ. Table 1 shows the parameter sets of the 5 species.

Figure 2 shows the experimental data for the mice, flies, maize, Tradescantia and

\footnotetext{
${ }^{1}$ In reality, geometric parameter $m$ varies due to body length and weight, so the growth curve of an animal is determined empirically. http://en.wikipedia.org/wiki/Generalised_logistic_function
} 
chrysanthemum along with the theoretical curve.

Figure 2 shows an cross-species experimental result and indicates that the predicted value based on our model matches well with the distribution of the experimental data. The time scale of the initiation of repair depends on the species and dose rate. However, expressing the dependency on species parameters and dose rate by a dimensionless time $\tau$ enabled a unified picture to be drawn.

Table 1 Parameter set for each species

\begin{tabular}{|c|c|c|c|c|c|c|}
\hline & & Mice & Dros-ophila & Maize & $\begin{array}{l}\text { Crysan- } \\
\text { themum }\end{array}$ & Tradescantia \\
\hline$\alpha$ & {$[1 / \mathrm{hr}]$} & $3.00 \mathrm{E}-03$ & $3.00 \mathrm{E}-04$ & $1.78 \mathrm{E}-01$ & 4.49E-03 & 6.94E-01 \\
\hline$\beta$ & {$[1 / \mathrm{Gy}]$} & $1.36 \mathrm{E}-01$ & $0.00 \mathrm{E}+00$ & $0.00 \mathrm{E}+00$ & $0.00 \mathrm{E}+00$ & $1.61 \mathrm{E}-01$ \\
\hline$\gamma$ & [1/Gy] & $2.94 \mathrm{E}-05$ & $2.32 \mathrm{E}-03$ & $2.02 \mathrm{E}-03$ & $6.49 \mathrm{E}-03$ & $1.63 \mathrm{E}-01$ \\
\hline$\kappa$ & {$[1 / \mathrm{hr}]$} & $3.24 \mathrm{E}-08$ & $0.00 \mathrm{E}+00$ & $0.00 \mathrm{E}+00$ & $0.00 \mathrm{E}+00$ & $2.91 \mathrm{E}-02$ \\
\hline
\end{tabular}

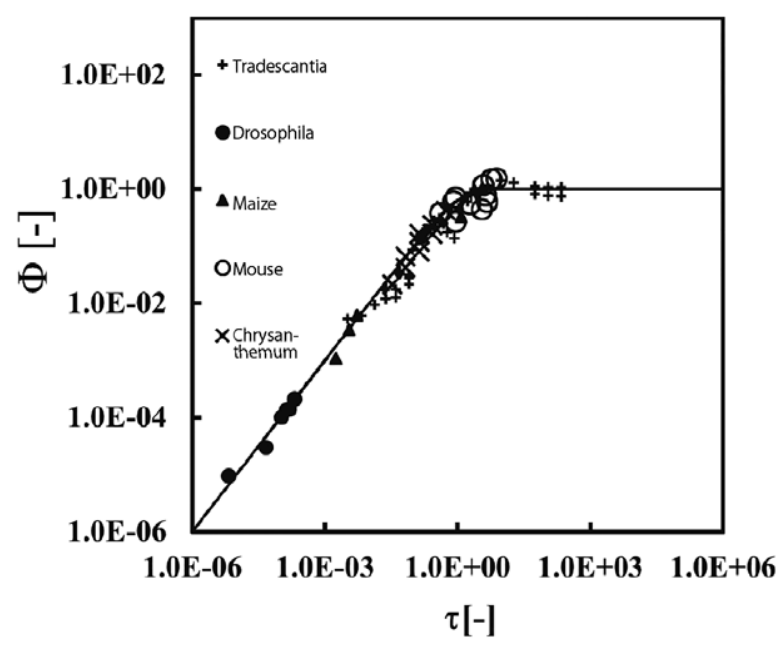

Figure 2 Reproduction of experimental data by scale function Vertical axis: value of screening function (common to organisms), Horizontal axis: dimensionless time.

\section{Spontaneous mutation}

We will now discuss the effect of mutation frequency on control data, i.e., without artificial irradiation. We focus on the parameters of the mouse, which is the closest to a human in terms of such modeling. Russell mentioned that the dose necessary to cause humans to have the same mutation rate as mice is $1 / 2$ of that required for mice; that is, humans are more receptive to such a dose ${ }^{2)}$. Note that this comparison is for reference purposes only. Organisms repeat metabolism and self-proliferation through interactions with the environment; such activities are one of the stimulations that occur in organisms. In fact, reactive oxygen species produced from such activities can damage DNA and cause replication error. We consider spontaneous mutation, which occurs because of stimulations other than artificial ones, as an equivalent stimulation and define 
$\kappa=\gamma d_{e f f}$

We can then obtain the following from the mouse data ${ }^{6}$.

$d_{\text {eff }}=1.11 \times 10^{-3} \mathrm{~Gy} / \mathrm{hr}$

Compared to the current dose rate in Fukushima, which is of the order of $\mu \mathrm{Sv} / \mathrm{hr}(\approx 1 \mu \mathrm{Gy} /$ hr), it is 1,000 times as high and does not even compare to the global average natural dose rate of $2.74 \times 10^{-7} \mathrm{~Gy} / \mathrm{hr}(2.4 \mathrm{mGy} / \mathrm{yr})$. According to the analysis by Tubiana et al., the equivalent dose rate for a spontaneous mutation rate in humans is $8.4 \mathrm{mGy} / \mathrm{h}$, which is consistent with the value for mice ${ }^{12}$.

\section{Summary}

This article introduced recovery mechanisms such as cell proliferation and apoptosis as well as the reparing function; death of normal cells and changes to mutated cells caused by radiation; and the "Whack-A-Mole model of destruction and repairing" for normal cells and mutated cells, with consideration for the death of mutated cells by radiation. Studying the functions of organisms means recognizing their strength for survival. LNT is only established when the recovery functions are weak. The dose rate effect could be observed for drosophila if we had low-dose data. With a high dose rate, repair could not catch up in a short time, and mutated cells rapidly increased, showing the symptoms of LNT in mice.

If we can understand the effect of radiation on organisms in a unified manner with the scaling rule, it will become the basis for estimating the same effect on humans. We can go beyond the qualitative assessment and possibly make a quantitative assessment. This will need to be verified through the use of epidemiology, biological experiments, and physical experiments.

After the Fukushima Daiichi Nuclear Accident, the "Committee on low-dose radiation effects" was established, wherein physicists and biologists engaged in heated debates. Bando used her experience in traffic flow theory to start the study, and Manabe, Kento Ichikawa, and Nakamura joined the research. We wanted to somehow quantify the effect of radiation by utilizing mathematical models and saw a hint in equations given in the "voices of the members" (Takashi Inamura: Journal of Physical Society of Japan, November 2011) ; on the basis of these factors, we developed this model. Later, Tsunoyama and Nakajima, with their expertise in plants and animals, joined, creating an interdisciplinary network. We received support from numerous individuals; from biology: Mitsuyuki Abe, Hiroshi Utsumi, Kazuko Uno, Shunichi Takeda, Ohtsura Niwa, Michiaki Kai; from the American Physical Society: Hanna M. Dobrovolny and Michael Ellis; and for advice on mathematical calculation: Takuya Matsuda and Hiroshi Isaka et al.

We held discussions across fields such as radiation biology, medicine, physics, and information science. This effort convinced us that serious exchange among diverse fields can lead to a new, fused field. The Fukushima Daiichi Nuclear Accident was no doubt a severe experience, but it served as an opportunity to re-examine the meaning of science. We wish to continue this pioneering work and expand our knowledge in new areas. We hope that doing so is a scientists'way of contributing to the rehabilitation of Fukushima. 


\section{Acknowledgment}

This research was partly supported by the Toyota Physical and Chemical Research Institute Scholars in 2014.

Added entries by author 24, July, 2018.

The later development of this research and its future prospects were presented at the "International Workshop on the Biological Effects of Radiation -bridging the gap between radiobiology and medical use of ionizing radiation-" (HP: https://www.rcnp.osaka-u.ac. jp/ ber2018/), which was held at the Osaka University Nakanoshima Center in March 2018. The content of this presentation is scheduled to be published in a Special Issue of the International Journal of Radiation Biology.

The other research activities of our group are published at https://www.rcnp.osaka-u.ac. jp/ manabe/project.html.

Moreover, this research requires interdisciplinary cooperation. Thus, we established Committees for Research Promotion in Specialized Areas on "multidisciplinary research on biological effects of radiation" at Japan Society for the Promotion of Science in October 2015, with Dr. Takahiro Wada from Kansai University as the chairperson. This committee supports multidisciplinary research based on purely scientific discussions, and its objective is to construct a common platform for promoting comprehensive studies on the impact of radiation through active exchange of opinions between the industry and academia. Though it is planned to cease operations in September 2018, we are currently exploring ways of continuing such activities. The aforementioned international conference was hosted by our committee, and we decided to work toward the construction of a platform for "multidisciplinary research on biological effects of radiation."

\section{References}

1) Muller HJ. Science. 1927; 66:84.

2) Kelly EM, Russell WL. Proc. Natl. Acad. Sci. 1982; 7(92) :542.

3) Douglas LE. Actions of Radiations on Living Cells. Cambridge University Press, 1954.

4) Chadwick KH, Leenhouts HP. Phys. Med. Biol. 1973 1(81):78.

5) Kellerer AM, Rossi HH. Radiat. Res. 1978; 75(3) :471.

6) Manabe Y, Ichikawa K, Bando M. J. Phys. Soc. Jpn. 2012; 81:104004. Manabe Y, Bando M. J. Phys. Soc. Jpn. 2013; 82:094004.

Manabe Y, Nakamura I, Bando M. J. Phys. Soc. Jpn. 2014 accepted.

7) Richards FJ. J. Exp. Bot. 1959; 10(2) : 290.

8) Spencer WP, Stern C. Genetics. 1948; 33(1):43.

9) Mabuchi T, Matsumura S. Jpn. J. Genet. 1964; 39:131.

10) Sparrow AH, Underbrink AG, Rossi HH. Science. 1972; 176(4037):916 Nauman HC, Underbrink AG, Sparrow AH. Radiat. Res. 1975; 62:79.

11) Yamaguchi H, Shimizu A, Degi K, Morishita T. Breeding Sci. 2008; 58(3) :331.

12) Tubiana M, Feinendegen LE, Kaminski JM. Radiology. 2009; 251(1):13. 